\title{
On Homomorphisms Between Generalized Polygons
}

\author{
Dedicated to Prof. Dr. H. R. Salzmann on the occasion of his \\ 65th anniversary
}

\author{
RICHARD BÖDI and LINUS KRAMER \\ Mathematisches Institut, Universität Tübingen, Auf der Morgenstelle 10, 72076 Tübingen, \\ Germany.e-mail: mmisa01@mailserv.zdv.uni-tuebingen.de
}

(Received: 22 February 1994)

\begin{abstract}
We consider homomorphisms between abstract, topological, and smooth generalized polygons. It is shown that a continuous homomorphism is either injective or locally constant. A continuous homomorphism between smooth generalized polygons is always a smooth embedding. We apply this result to isoparametric submanifolds.
\end{abstract}

Mathematics Subject Classifications (1991): 51A25, 51E24, 51H20, 51H25.

The aim of this paper is to investigate homomorphisms of abstract, topological, and smooth generalized polygons. The first two sections deal with generalized polygons and their homomorphisms. We have tried to make this paper self-contained, so we give a short exposition of the coordinatization and the algebraic operations $(+,-, \bullet, /)$ of a generalized polygon. The most important theorems at this stage are the characterization (2.7) of injective homomorphisms, and the proof (2.9) of Pasini's theorem [27] that the fibers of a non-injective homomorphism are infinite.

Topological polygons are introduced in Section 3. Here, the main result is that a homomorphism is either injective or locally constant (3.4); in particular, a connected polygon admits only injective homomorphisms. For topological projective planes, this has been proved by Breitsprecher [4]. This result may be compared to Pasini's theorem [27] that finite polygons admit only injective homomorphisms.

In the last section, we introduce smooth polygons. The main result of this paper states that a continuous homomorphism between smooth polygons is always a smooth embedding (4.7). In particular, every continuous automorphism of a smooth polygon is smooth, and thus the topological automorphism group of the polygon, endowed with the compact-open topology, is a smooth Lie transformation group (4.9). As an application, we prove a strong inhomogeneity theorem for a class of isoparametric hypersurfaces. 


\section{Generalized Polygons}

(1.1) DEFINITION. An incidence structure $\mathfrak{P}=(\mathcal{P}, \mathcal{L}, \mathcal{F})$ is a triple, consisting of a set $\mathcal{P}$ of points, a set $\mathcal{L}$ of lines, and a set $\mathcal{F} \subseteq \mathcal{P} \times \mathcal{L}$ of flags. We say that a point $p$ is incident with a line $\ell$, if $(p, \ell)$ is a flag. The union $\mathcal{V}=\mathcal{P} \cup \mathcal{L}$ is called the set of vertices of $\mathfrak{P}$ (we assume that $\mathcal{P} \cap \mathcal{L}=\emptyset$ ). For a vertex $x$, we put $\mathcal{V}_{x}=\{y \in \mathcal{V} \mid y$ is incident with $x\}$ (Knarr [21] denotes this set by $\Gamma(x)$ ).

A point row $\mathcal{V}_{\ell}=L \subseteq \mathcal{P}$ is the set of all points that are incident with a certain line $\ell$. Similarly, a pencil of lines $\mathcal{V}_{p}=\mathcal{L}_{p} \subseteq \mathcal{L}$ is the set of all lines that are incident with a certain point $p$.

A $k$-chain is a sequence $\mathbf{v}=\left(v_{0}, v_{1}, \ldots, v_{k}\right) \in \mathcal{V}^{k+1}$ of vertices with the property that $v_{i}$ is incident with $v_{i+1}$ for $0 \leq i<k$, and with $v_{i} \neq v_{i+2}$ for $0 \leq i<k-1$; we say that the $k$-chain $\mathbf{v}$ joins $v_{0}$ and $v_{k}$ (note that we exclude stammering chains). If two vertices $x, y$ can be joined by a $k$-chain, but not by any $k^{\prime}$-chain for $k^{\prime}<k$, we say that the distance $d(x, y)$ between $x$ and $y$ is $k$.

An ordinary $k$-gon is a $2 k$-chain $\left(v_{0}, v_{1}, \ldots, v_{2 k-1}, v_{0}\right)$ with the property that $v_{i} \neq v_{j}$ for $0 \leq i<j<2 k$.

(1.2) DEFINITION. Let $n \geq 3$ be an integer. A generalized $n$-gon is an incidence structure $\mathfrak{P}=(\mathcal{P}, \mathcal{L}, \mathcal{F})$ with the following properties:

$\left.(n \text {-Gon })_{1}\right)$ There are no ordinary $k$-gons for $2 \leq k<n$.

$\left(n-\mathrm{Gon}_{2}\right)$ Any two vertices are contained in some ordinary $n$-gon.

$\left.(n \text {-Gon })_{3}\right)$ There exists an ordinary $(n+1)$-gon.

Let $v_{0}, v_{k}$ be two vertices with distance $d\left(v_{0}, v_{k}\right)=k<n$. Condition $\left(n\right.$-Gon $\left.{ }_{1}\right)$ says that the $k$-chain $\left(v_{0}, \ldots, v_{k-1}, v_{k}\right)$ joining $v_{0}$ and $v_{k}$ is uniquely determined by the vertices $v_{0}, v_{k}$. Hence we may define a map $f_{k}\left(v_{0}, v_{k}\right)=v_{k-1}$. Condition $\left(n-\mathrm{Gon}_{3}\right)$ is equivalent to the following condition, see Schroth [34, 2.6], e.g.

$\left(n\right.$-Gon $\left.{ }_{3}^{\prime}\right)$ Every point row and every pencil of lines contains at least three vertices.

Note that the definition of a generalized polygon is self-dual: $(\mathcal{P}, \mathcal{L}, \mathcal{F})$ is a generalized polygon if and only if the dual $\left(\mathcal{L}, \mathcal{P}, \mathcal{F}^{-1}\right)$ is a generalized polygon. For this reason, we will sometimes give a proof only for the point space; the corresponding statement for the line space follows by duality.

(1.3) EXAMPLE. Let $\mathfrak{P}=(\mathcal{P}, \mathcal{L}, \mathcal{F})$ be a generalized triangle. The conditions $(n$-Gon 1$)$ and $(n \text {-Gon })_{2}$ say that any two different points $p, q$ can be joined by a unique line $p \vee q=f_{2}(p, q)$, and that any two different lines $\ell, h$ intersect in a unique point $\ell \wedge h=f_{2}(\ell, h)$. Thus, the generalized triangles are precisely the projective planes.

The following lemma will be required frequently.

(1.4) LEMMA (cp. Tits $[40,3.30])$. Let $x, y \in \mathcal{V}$ be two vertices of the same type 
(i.e. $x$ and $y$ are both points or $x$ and $y$ are both lines) in a generalized n-gon $\mathfrak{P}$. Then there exists a vertex $z \in \mathcal{V}$ with $d(x, z)=d(y, z)=n$.

Proof. Let $z$ be a vertex with $d(x, z)=n$ that has maximal distance $k$ to $y$, and suppose that $k \neq n$. Thus $k \leq n-2$. Choose a vertex $b \in \mathcal{V}_{z}-\left\{f_{k}(y, z)\right\}$. We have $d(b, x)=n-1$ and $d(b, y)=k+1$, hence there is an element $z^{\prime} \in$ $\mathcal{V}_{b}-\left\{f_{n-1}(x, b), f_{k+1}(y, b)\right\}$. But now $d\left(z^{\prime}, y\right)=k+2$ and $d\left(z^{\prime}, x\right)=n$, a contradiction to the maximality of $k$.

(1.5) DEFINITION. Let $\mathfrak{P}=(\mathcal{P}, \mathcal{L}, \mathcal{F})$ be a generalized $n$-gon, and let $x, y$ be two vertices of maximum distance $d(x, y)=n$. We define a perspectivity $[y, x]: \mathcal{V}_{x} \rightarrow \mathcal{V}_{y}$ by $v \mapsto f_{n-1}(v, y)$, cp. Knarr [21]. Clearly, $[x, y][y, x]=\mathrm{id} \mathcal{V}_{x}$. A concatenation of perspectivities is called a projectivity; we put $[z, y][y, x]=$ $[z, y, x]$, etc. The collection of all projectivities of $\mathfrak{P}$ forms a groupoid. If we fix a vertex $x$, then the group $\Pi(x)$ of all projectivities from $\mathcal{V}_{x}$ to $\mathcal{V}_{x}$ is two-transitive on $\mathcal{V}_{x}$ (see $(1.8)$ or Knarr $[21,1.2]$ ).

By (1.4), projectivities exist between any two vertices of the same type; if $n$ is odd, then projectivities exist between any two vertices.

We shall make use of the following coordinatization of the point space.

(1.6) DEFINITION. Let $\mathfrak{P}=(\mathcal{P}, \mathcal{L}, \mathcal{F})$ be a generalized $n$-gon. Let $v$ be a vertex such that the vertices $q$ with distance $d(v, q)=n-1$ are points (i.e. $v$ is a point if $n$ is odd, and a line if $n$ is even), and let $u$ be a vertex incident with $v$. We put

$$
\mathcal{P}_{n-1}(u, v)=\left\{q \in \mathcal{P} \mid d(q, v)=n-1 \text { and } f_{n-1}(q, v) \neq u\right\} .
$$

This set is called the big cell in $\mathcal{P}$ with respect to $(u, v)$. In the case of a projective plane, $\mathcal{P}_{2}(\ell, p)=\mathcal{P}-L$ is an affine plane.

Now choose an $n$-chain $\mathbf{v}=\left(v_{0}=v, v_{1}=u, v_{2}, \ldots, v_{n}\right)$. For $x \in \mathcal{P}_{n-1}(u, v)$ let $\left(x_{0}=v, x_{1}, \ldots, x_{n-1}=x\right)$ denote the unique $(n-1)$-chain from $v$ to $x$. Since $d\left(x_{i}, v_{n-i}\right)=n$, we have $d\left(x_{i+1}, v_{n-i}\right)=n-1$, and we may define $y_{i}=f_{n-1}\left(x_{i+1}, v_{n-i}\right)$ for $0 \leq i<n-1$. Then we have $x_{i+1}=f_{n-1}\left(y_{i}, x_{i}\right)$. Note also that $x_{i}=f_{n-1}\left(v_{n-i-2}, x_{i+1}\right)$. Therefore, we get a bijection

$$
\begin{aligned}
\xi_{\mathrm{v}} & : \mathcal{P}_{n-1}(u, v) \rightarrow\left(\mathcal{V}_{v_{n}}-\left\{v_{n-1}\right\}\right) \\
& \times\left(\mathcal{V}_{v_{n-1}}-\left\{v_{n-2}\right\}\right) \times \cdots \times\left(\mathcal{V}_{v_{2}}-\left\{v_{1}\right\}\right): x \mapsto\left(y_{0}, \ldots, y_{n-2}\right)
\end{aligned}
$$

which can be expressed in both directions in terms of the function $f_{n-1}$ (cp. Knarr $[22,2.5])$. There, the set $\mathcal{P}_{n-1}(u, v)$ is denoted by $\Gamma_{n-1}(v, u)$.

(1.7) DEFINITION. Let $\mathfrak{P}=(\mathcal{P}, \mathcal{L}, \mathcal{F})$ be a generalized $n$-gon. Let $\mathbf{v}=\left(v_{0}, \ldots\right.$, $\left.v_{2 n-1}, v_{0}\right)$ be an ordinary $n$-gon. We put $0_{K}=v_{1}, \infty_{K}=v_{2 n-1}, 0_{L}=v_{n-2}$, and $\infty_{L}=v_{n}$. Finally, we set $L=\mathcal{V}_{v_{n-1}}-\left\{\infty_{L}\right\}$ and $K=\mathcal{V}_{v_{0}}-\left\{\infty_{K}\right\}$, and we choose an element $1_{L} \in L-\left\{0_{L}\right\}$. For $(x, y) \in K \times L$ we define

$$
x \bullet y=f_{n-1}\left(f_{n-1}\left(f_{n-1}\left(f_{n-1}\left(x, 1_{L}\right), v_{2 n-2}\right), y\right), v_{0}\right) \in K .
$$


Similarly, for $(x, y) \in K \times\left(L-\left\{0_{L}\right\}\right)$, we define

$$
x / y=f_{n-1}\left(f_{n-1}\left(f_{n-1}\left(f_{n-1}(x, y), v_{2 n-2}\right), 1_{L}\right), v_{0}\right) \in K .
$$

It is readily verified that $x \mapsto x \bullet y$ is a projectivity for $y \neq 0_{L}$, with inverse $x \mapsto x / y$. We leave it to the reader to check that the expressions on the right-hand side are well defined. These maps have the following properties:

(1) $x \bullet 0_{L}=0_{K}=0_{K} \bullet y$

(2) $x \bullet 1_{L}=x$

(3) $(x \bullet y) / y=(x / y) \bullet y=x$ for $y \neq 0_{L}$.

The map $\bullet$ is called the multiplication with respect to $\left(\mathbf{v}, 1_{L}\right)$, cp. Grundhöfer $e t$ $a l$. [15, 1.1], and Hanssens and Van Maldeghem [18] for $n=4$.

(1.8) DEFINITION. Let $\mathfrak{P}=(\mathcal{P}, \mathcal{L}, \mathcal{F})$ be a generalized $n$-gon. Let $\mathbf{v}=\left(v_{0}, \ldots\right.$, $\left.v_{2 n-1}, v_{0}\right)$ be an ordinary $n$-gon. We put $0_{K}=v_{1}, \infty_{K}=v_{2 n-1}$, and $K=$ $\mathcal{V}_{v_{0}}-\left\{\infty_{K}\right\}$. Furthermore, we choose an element $a \in \mathcal{V}_{v_{n+1}}-\left\{v_{n}, v_{n+2}\right\}$. For $y \in K$, we consider the projectivity

$$
\pi_{y}=\left[v_{0}, v_{n}, f_{n-1}(a, y), v_{n+2}, f_{n-1}\left(a, v_{1}\right), v_{n}, v_{0}\right] \in \Pi\left(v_{0}\right) .
$$

Note that $\pi_{y}\left(\infty_{K}\right)=\infty_{K}, \pi_{y}\left(0_{K}\right)=y$, and $\pi_{0_{K}}=\mathrm{id} v_{v_{0}}$. Thus we may define maps $\pm: K \times K \rightarrow K$ by $x+y=\pi_{y}(x)$ and by $x-y=\pi_{y}^{-1}(x)$. Then we get the identities

(1) $x+0_{K}=0_{K}+x=x$

(2) $(x+y)-y=(x-y)+y=x$.

Therefore, $\left(K, 0_{K},+\right)$ is a right loop in the sense of [3]. The map + is called the addition with respect to $(\mathrm{v}, a)$, cp. Grundhöfer et al. [15, 1.4], and Hanssens and Van Maldeghem [18] for $n=4$.

\section{Homomorphisms Between Generalized Polygons}

(2.1) DEFINITION. Let $\mathfrak{P}=(\mathcal{P}, \mathcal{L}, \mathcal{F})$ and $\mathfrak{P}^{\prime}=\left(\mathcal{P}^{\prime}, \mathcal{L}^{\prime}, \mathcal{F}^{\prime}\right)$ be generalized $n$-gons. A homomorphism $\varphi: \mathfrak{P} \rightarrow \mathfrak{P}^{\prime}$ is a map $\varphi: \mathcal{V} \rightarrow \mathcal{V}^{\prime}$ with the following properties:

$\left(\mathrm{Hom}_{1}\right)$ Points are mapped to points, lines are mapped to lines, and flags are mapped to flags.

$\left(\operatorname{Hom}_{2}\right)$ The image $\varphi(\mathfrak{P})=(\varphi(\mathcal{P}), \varphi(\mathcal{L}),(\varphi \times \varphi)(\mathcal{F}))$ contains an ordinary $(n+1)$-gon.

The second condition guarantees that the image of a generalized $n$-gon is again a generalized $n$-gon. Note, however, that a homomorphism may diminish the distance between vertices. 
(2.2) EXAMPLE. Let $\mathbb{Q}_{p}$ denote the field of $p$-adic numbers and let $f: \mathbb{Q}_{p} \rightarrow \mathbb{F}_{p}$ be the canonical epimorphism onto the residue class field $\mathbb{F}_{p}$. This epimorphism induces an epimorphism between the associated projective planes over $\mathbb{Q}_{p}$ and $\mathbb{F}_{p}$, respectively (compare Prieß-Crampe [28, V, Section 4]).

For the remainder of this section, let $\varphi: \mathfrak{P} \rightarrow \mathfrak{P}^{\prime}$ be a homomorphism between generalized $n$-gons $\mathfrak{P}$ and $\mathfrak{P}^{\prime}$. We denote the distance functions of $\mathfrak{P}$ and $\mathfrak{P}^{\prime}$ by $d$ and $d^{\prime}$, respectively.

(2.3) LEMMA. Let $d^{\varphi}$ denote the distance function in the image $\varphi(\mathfrak{P})$. Then $d^{\prime}=d^{\varphi}$. In particular, two images $\varphi(p), \varphi(\ell)$ are incident in $\mathfrak{P}^{\prime}$ if and only if there is a flag $(q, h) \in \mathcal{F}$ with $(\varphi(q), \varphi(h))=(\varphi(p), \varphi(\ell))$.

Proof. Suppose that $d^{\varphi}\left(v_{0}, v_{k}\right)=k>d^{\prime}\left(v_{0}, v_{k}\right)=k^{\prime}$. Let $\left(v_{0}, \ldots, v_{k}\right)$ be a minimal $k$-chain in $\varphi(\mathfrak{P})$, and let $\left(v_{0}, w_{1}, \ldots, w_{k^{\prime}-1}, v_{k}\right)$ be a minimal $k^{\prime}$-chain in $\mathfrak{P}^{\prime}$. Deleting the repetitions in $\left(v_{0}, \ldots, v_{k}, w_{k^{\prime}-1}, \ldots, w_{1}, v_{0}\right)$, we get an ordinary $m$-gon in $\mathfrak{P}^{\prime}$ for some $2 \leq m \leq\left(k+k^{\prime}\right) / 2<n$, a contradiction.

The following lemma, which has a number of immediate consequences, is based on a simple, but useful observation.

(2.4) LEMMA. If $d^{\prime}(\varphi(x), \varphi(y))=n$, then $d(x, y)=n$, and $\varphi \circ[x, y]=$ $[\varphi(x), \varphi(y)] \circ \varphi$. If $d^{\prime}(\varphi(x), \varphi(y))=n-1$, then $d(x, y)=n-1$, and we have $\varphi\left(f_{n-1}(x, y)\right)=f_{n-1}^{\prime}(\varphi(x), \varphi(y))$.

Proof. This is clear from $d^{\prime}(\varphi(x), \varphi(y)) \leq d(x, y)$.

(2.5) COROLLARY (cp. Pasini [27, Lemma 1]). For every $x \in \mathcal{V}$, the map $\varphi \mid \mathcal{V}_{x}: \mathcal{V}_{x} \rightarrow \mathcal{V}_{\varphi(x)}^{\prime} \cap \varphi(\mathcal{V})$ is a surjection. In particular, the restriction $\varphi \mid \mathcal{V}_{x}$ assumes at least three different values.

Proof. Let $\varphi\left(x_{1}\right) \in \mathcal{V}_{\varphi(x)}^{\prime}$. Choose an $(n-1)$-chain $\left(\varphi(x), \varphi\left(x_{1}\right), \ldots, \varphi\left(x_{n-1}\right)\right)$ in $\mathfrak{P}^{\prime}$. Now $d\left(x, x_{n-1}\right)=n-1$ by $(2.4)$, and $\varphi\left(f_{n-1}\left(x_{n-1}, x\right)\right)=\varphi\left(x_{1}\right)$.

(2.6) COROLLARY. Let $v_{n-1}$ be a vertex. Choose elements $v_{n}, v_{n-2}, 1_{L} \in \mathcal{V}_{v_{n-1}}$ with pairwise different images under $\varphi$. Choose a vertex $v_{2 n-1}$ with $d^{\prime}\left(\varphi\left(v_{n-1}\right)\right.$, $\left.\varphi\left(v_{2 n-1}\right)\right)=n$. This uniquely determines an ordinary $n$-gon $\mathbf{v}=\left(v_{0}, \ldots, v_{2 n-1}, v_{0}\right)$ in $\mathfrak{P}$, and the image of $\mathbf{v}$ is an ordinary $n$-gon in $\mathfrak{P}^{\prime}$ (because $\varphi\left(v_{n}\right) \neq \varphi\left(v_{n-2}\right)$ ). Thus we may consider the multiplication $\bullet$ in $\mathfrak{P}$ with respect to $\left(\mathbf{v}, 1_{L}\right)$, and the multiplication $\bullet^{\prime}$ in $\mathfrak{P}^{\prime}$ with respect to $\left(\varphi(\mathbf{v}), \varphi\left(1_{L}\right)\right)$. Provided that $\varphi(x) \neq \varphi\left(\infty_{K}\right)$ and $\varphi(y) \neq \varphi\left(\infty_{L}\right)$, we get the relation $\varphi(x \bullet y)=\varphi(x) \bullet^{\prime} \varphi(y)$.

(2.7) COROLLARY. Let $v, v_{n-1} \in \mathcal{V}$ be two vertices with $d^{\prime}\left(\varphi(v), \varphi\left(v_{n-1}\right)\right)=$ $n-1$. Put $u=f_{n-1}\left(v_{n-1}, v\right)$ and consider the big cells $\mathcal{P}_{n-1}(u, v) \subseteq \mathcal{P}$ and $\mathcal{P}_{n-1}^{\prime}(\varphi(u), \varphi(v)) \subseteq \mathcal{P}^{\prime}$ as defined in (1.6). Let $\mathbf{v}$ and $\mathbf{v}^{\prime}$ denote the $(n-1)$-chains determined by $\left(v, v_{n-1}\right)$ and $\left(\varphi(v), \varphi\left(v_{n-1}\right)\right)$, respectively. Setting

$$
U=\varphi^{-1}\left(\mathcal{P}_{n-1}^{\prime}(\varphi(u), \varphi(v))\right) \subseteq \mathcal{P}_{n-1}(u, v)
$$


we get the relation

$$
\varphi \mid U=\xi_{\mathbf{V}^{\prime}}^{-1} \circ\left(\left(\varphi \mid \mathcal{V}_{v_{n}}-\left\{v_{n-1}\right\}\right) \times \cdots \times\left(\varphi \mid \mathcal{V}_{v_{2}}-\left\{v_{1}\right\}\right)\right) \circ \xi_{\mathbf{v}}
$$

The next theorem gives a local characterization of injective homomorphisms.

(2.8) THEOREM. Let $\varphi: \mathfrak{P} \rightarrow \mathfrak{P}^{\prime}$ be a homomorphism between generalized $n$ gons. Let $x$ be a vertex of $\mathfrak{P}$. If the restriction $\varphi \mid \mathcal{V}_{x}$ is injective, then $\varphi$ is injective on $\mathcal{V}$, that is, $\varphi$ is an embedding of generalized $n$-gons.

Proof. Suppose that $y \in \mathcal{V}$ is a vertex of the same type as $x$. Pick a vertex $z$ with $d^{\prime}(\varphi(x), \varphi(z))=d^{\prime}(\varphi(y), \varphi(z))=n$. Then the projectivity $[y, z, x, z, y]=$

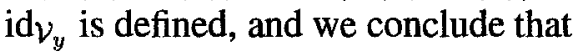

$$
\varphi \mid \mathcal{V}_{y}=[\varphi(y), \varphi(z), \varphi(x)] \circ\left(\varphi \mid \mathcal{V}_{x}\right) \circ[x, z, y]
$$

and

$$
\varphi \mid \mathcal{V}_{z}=[\varphi(z), \varphi(x)] \circ\left(\varphi \mid \mathcal{V}_{x}\right) \circ[x, z]
$$

This shows that if $\varphi$ is injective on $\mathcal{V}_{x}$, then it is injective on both $\mathcal{V}_{y}$ and $\mathcal{V}_{z}$.

Thus, if $n$ is odd, then the restriction of $\varphi$ to any point row and to any pencil of lines is injective.

Next, suppose that $n$ is even, and that $\varphi \mid \mathcal{V}_{x}$ is not injective. Suppose that $\varphi(b)=\varphi\left(0_{L}\right)$ for two vertices $b, 0_{L} \in \mathcal{V}_{x}$. Choose two more elements $\infty_{L}, 1_{L} \epsilon$ $\mathcal{V}_{x}$ with different $\varphi$-images. Put $v_{n-2}=0_{L}, v_{n-1}=x, v_{n}=\infty_{L}$, and choose a vertex $v_{2 n-1}=0_{K}$ with $d^{\prime}\left(\varphi\left(v_{n-1}\right), \varphi\left(v_{2 n-1}\right)\right)=n$. This determines a unique ordinary $n$-gon $\left(v_{0}, \ldots, v_{2 n-1}, v_{0}\right)$, and we may apply the multiplication map of (2.6) to show that $\varphi$ is not injective on $K \cup\left\{\infty_{K}\right\}=\mathcal{V}_{v_{0}}$. So let $u \in K-\left\{0_{K}\right\}$. If $\varphi(u)=\varphi\left(\infty_{K}\right)$, then we are done. Otherwise, we have the relation $\varphi(u \bullet b)=$ $\varphi(u) \bullet^{\prime} \varphi(b)=\varphi\left(0_{K}\right)$, but $u \bullet b \neq 0_{K}$. Hence $\varphi$ is not injective on $K \cup\left\{\infty_{K}\right\}$.

Finally, assume that $\varphi$ is not injective on $\mathcal{V}$. By what we have proved so far, we have to find two different vertices $x, y$ of distance 2 with $\varphi(x)=\varphi(y)$. To that end, we choose two such vertices with minimal distance $d(x, y)=k$. We claim that $k=2$. Otherwise, choose a minimal $k$-chain $\left(x, v_{1}, \ldots, v_{k-1}, y\right)$. Now the restriction $\varphi \mid\left\{v_{1}, v_{2}, \ldots, y\right\}$ cannot be injective, because otherwise the image $\varphi(\mathfrak{P})$ would contain an ordinary $k / 2$-gon. But the distance between the $k$ elements $\left\{v_{1}, v_{2}, \ldots, y\right\}$ is strictly less then $k$, a contradiction.

The next lemma, which states that the fibers of $\varphi$ in a point row or in a pencil of lines are isomorphic via projectivities, leads to a simple proof of Pasini's theorem.

(2.9) LEMMA. Let $a, b$ be vertices of $\mathfrak{P}$. In case that $n$ is even, we assume that $a$ and $b$ have the same type. If $n$ is odd, then $a, b$ may be arbitrary vertices. Let $x \in \mathcal{V}_{a}$ and $y \in \mathcal{V}_{b}$, and put $X=\left\{z \in \mathcal{V}_{a} \mid \varphi(z)=\varphi(x)\right\}$ and $Y=\left\{z \in \mathcal{V}_{b} \mid \varphi(z)=\varphi(y)\right\}$. Then there exists a projectivity $\pi$ from a to $b$ with $\pi(X)=Y$. 
Proof. Let $\pi^{\prime}=\left[\varphi(b), \varphi\left(v_{1}\right), \ldots, \varphi\left(v_{k}\right), \varphi(a)\right]$ be a projectivity in $\mathfrak{P}^{\prime}$ from $\varphi(a)$ to $\varphi(b)$ with $\pi^{\prime}(\varphi(x))=\varphi(y)$. Note that by the assumptions, projectivities between $\varphi(a)$ and $\varphi(b)$ exist, and that $\mathcal{V}_{\varphi(b)}^{\prime}$ is homogeneous under $\Pi(\varphi(b))$. Thus a projectivity $\pi^{\prime}$ with the property stated above exists.

By (2.4) we get a projectivity $\pi=\left[b, v_{1}, \ldots, v_{k}, a\right]$ in $\mathfrak{P}$ from $a$ to $b$. Now for $z \in X$, we have $\varphi(\pi(z))=\pi^{\prime}(\varphi(z))=\varphi(y)$, hence $\pi(X) \subseteq Y$. Conversely, if $\pi(z) \in Y$, then $\varphi(y)=\varphi(\pi(z))=\pi^{\prime}(\varphi(z))$, hence $\varphi(z)=\varphi(x)$, and the claim follows.

An immediate consequence is the following theorem, proved first by Pasini [27] (see also Dembowski [9], Hughes [20] and Mortimer [26] for $n=3$ ).

(2.10) THEOREM. Let $\varphi: \mathfrak{P} \rightarrow \mathfrak{P}^{\prime}$ be a non-injective homomorphism between generalized n-gons. Then for every pair of incident vertices $x, y \in \mathcal{V}$, the set $X=\left\{z \in \mathcal{V}_{y} \mid \varphi(z)=\varphi(x)\right\}$ is infinite. In particular, $\mathfrak{P}$ is infinite.

Proof. Let $a, b \in \mathcal{V}$ be vertices with $d^{\prime}(\varphi(a), \varphi(b))=n$. By (2.8), there are two vertices $x_{1}, y_{1} \in \mathcal{V}_{a}$ with $\varphi\left(x_{1}\right)=\varphi\left(x_{2}\right)$. Let $\left(a, x_{1}, x_{2}, \ldots, x_{n-1}, b\right)$ and $\left(a, y_{1}, y_{2}, \ldots, y_{n-1}, b\right)$ denote the corresponding $n$-chains. Clearly, we have $\varphi\left(x_{i}\right)=\varphi\left(y_{i}\right)$ for $1 \leq i<n$. We put $X=\left\{z \in \mathcal{V}_{x_{n-1}} \mid \varphi(z) \neq \varphi\left(x_{n-2}\right)\right\}$, $Y=\left\{z \in \mathcal{V}_{y_{1}} \mid \varphi(z)=\varphi\left(y_{2}\right)\right\}$, and $\pi=\left[y_{1}, x_{n-1}\right]$. We claim that $\pi(X) \subseteq Y$. Indeed, for $z \in \mathcal{V}_{x_{n-1}}$, the distance between $\varphi(z)$ and $\varphi(\pi(z))$ is $n-2$ or $n-4$ (because $d^{\prime}(\varphi(a), \varphi(b))=n$ ), and it is $n-4$ if and only if $\varphi(z)=\varphi\left(x_{n-2}\right)$ and $\varphi(\pi(z))=\varphi\left(y_{2}\right)$. Thus, if $z \in X$, then $\varphi(\pi(z))=\varphi\left(y_{2}\right)$. Now let $\alpha+1$ denote the cardinality of $\varphi\left(\mathcal{V}_{y_{1}}\right)$, and let $\eta$ denote the cardinality of $Y$. By (2.5), we have $\alpha \geq 2$. By (2.9), the cardinality of $X$ is $\alpha \eta$. Hence $\alpha \eta \leq \eta$, and thus $\eta$ is an infinite cardinal number. By (2.9), the cardinality $\eta$ of the fiber is independent of the vertices $y_{1}, y_{2}$ if $n$ is odd, and it depends only on the type of $y_{1}$ if $n$ is even.

\section{Topological Polygons and their Homomorphisms}

(3.1) DEFINITION. Let $\mathfrak{P}=(\mathcal{P}, \mathcal{L}, \mathcal{F})$ be a generalized $n$-gon. We say that $\mathfrak{P}$ is a topological n-gon, if $\mathcal{P}$ and $\mathcal{L}$ carry non-trivial (i.e. neither discrete nor antidiscrete) topologies such that the map $f_{n-1}$ is continuous on its domain $\{(x, y) \in$ $\mathcal{V} \times \mathcal{V} \mid d(x, y)=n-1\}$. Note that this implies that every projectivity is a homeomorphism.

Our notion of a topological $n$-gon agrees for $n=3$ with the usual definition of a topological projective plane, see, for example, Salzmann [30], [33], or Skornjakov [36]. For $n=4$, it agrees with the definitions of Forst [11] and Grundhöfer and Knarr [13] of a topological generalized quadrangle. It is stronger than the definition of Burns and Spatzier [7]; the continuity of $f_{n-1}$ implies that $\mathcal{F}$ is closed in $\mathcal{P} \times \mathcal{L}$, see [24], but the converse is not true, unless $\mathcal{P}$ and $\mathcal{L}$ are compact spaces (cp. Grundhöfer and Van Maldeghem [14, 2.1(a)]).

The following facts are proved in [24]. 
(a) Every point row and every pencil of lines is a doubly homogeneous, closed subspace of $\mathcal{P}$ and $\mathcal{L}$, respectively.

(b) The spaces $\mathcal{P}, \mathcal{L}$ and $\mathcal{F}$, as well as every point row and every pencil of lines are non-discrete Hausdorff spaces.

(c) The point rows are connected if and only if $\mathcal{P}, \mathcal{L}$, and $\mathcal{F}$ are connected.

(d) For every vertex $x$, the set $\{y \in \mathcal{V} \mid d(x, y)=n-1\}$ is open in $\mathcal{V}$. In particular, the big cell $\mathcal{P}_{n-1}(u, v)$ defined in (1.6) is open in $\mathcal{P}$. The map $\xi_{\mathrm{v}}$ is a homeomorphism.

(3.2) DEFINITION. A homomorphism $\varphi: \mathfrak{P} \rightarrow \mathfrak{P}^{\prime}$ between two topological $n$ gons is called continuous if $\varphi$ is continuous on $\mathcal{P}$ and on $\mathcal{L}$. Note that by (2.3), the image $\varphi(\mathfrak{P})$ is again a topological polygon.

(3.3) THEOREM. Let $\varphi$ be a continuous homomorphism between topological polygons $\mathfrak{P}$ and $\mathfrak{P}^{\prime}$. If $\varphi$ is not injective, then $\varphi$ is locally constant on each point row and on each pencil of lines. Hence if $\mathcal{P}$ is connected, then $\varphi$ is injective.

Proof. Suppose that $\varphi$ is not injective. Choose an ordinary $n$-gon $\mathbf{v}$ and a vertex $1_{L}$ as in (2.6), such that there is a vertex $b \in L-\left\{0_{L}\right\}$ with $\varphi(b)=\varphi\left(0_{L}\right)$. Consider the open set $V=\left\{x \in K \mid \varphi(x) \neq \varphi\left(\infty_{K}\right)\right\}$. Now $\varphi(V \bullet b)=\varphi(V) \bullet \varphi(b)=$ $\left\{\varphi\left(0_{K}\right)\right\}$, and therefore $\varphi$ is constant on the open set $V \bullet b \subseteq K$. By (2.9), every preimage of a vertex is open in $K \cup\left\{\infty_{K}\right\}$, hence $\varphi \mid K \cup\left\{\infty_{K}\right\}$ is locally constant. Therefore, there exists a point row and a pencil of lines where $\varphi$ is locally constant.

If $\varphi \mid V_{x}$ is locally constant, and if $y$ has the same type as $x$, then we find a vertex $z$ with $d^{\prime}(\varphi(x), \varphi(z))=d^{\prime}(\varphi(y), \varphi(z))=n$, and thus

$$
\varphi \mid \mathcal{V}_{y}=[\varphi(y), \varphi(z), \varphi(x)] \circ\left(\varphi \mid \mathcal{V}_{x}\right) \circ[x, z, y]
$$

is also locally constant.

(3.4) COROLLARY. Let $\varphi$ be a continuous homomorphism between topological polygons $\mathfrak{P}$ and $\mathfrak{P}^{\prime}$. If $\varphi$ is not injective, then $\varphi$ is locally constant on $\mathcal{P}$ and $\mathcal{L}$. (See Salzmann [31], Breitsprecher [4, 3.5], or Stroppel [38, Th. 6] for the case of topological projective planes.)

Proof. This follows from (3.3) and (2.7). Note that the set $U$ defined in (2.7) is open, and that every point is contained in a set of this type.

(3.5) PROPOSITION. Let $\varphi$ be an (abstract) homomorphism between topological polygons $\mathfrak{P}$ and $\mathfrak{P}^{\prime}$. If the restriction of $\varphi$ to some point row and to some pencil of lines is continuous, then $\varphi$ is continuous. If $n$ is odd, then it suffices that the restriction to some point row is continuous.

In particular, every root collineation (see, e.g., Ronan $[29, \mathrm{p} .66])$ of a topological polygon $\mathfrak{P}$ is a continuous automorphism. (See Breitsprecher $[4,1.2]$ for $n=3$ and Grundhöfer and Knarr $[13,5.1]$ for $n=4$.) 
Proof. Suppose that the restriction $\varphi \mid \mathcal{V}_{x}$ is continuous. If $y$ has the same type as $x$, then we may pick a vertex $z$ with $d(\varphi(z), \varphi(x))=d(\varphi(z), \varphi(y))=n$. Now the restrictions

$$
\varphi \mid \mathcal{V}_{y}=[\varphi(y), \varphi(z), \varphi(x)] \circ\left(\varphi \mid \mathcal{V}_{x}\right) \circ[x, z, y]
$$

and

$$
\varphi \mid \mathcal{V}_{z}=[\varphi(z), \varphi(x)] \circ\left(\varphi \mid \mathcal{V}_{x}\right) \circ[x, z]
$$

are continuous. Hence the restriction of $\varphi$ to any point row and any pencil of lines is continuous. By (2.7), the map $\varphi$ is continuous on the open set $U$ defined in (2.7). Since every point is contained in a set of this type, $\varphi$ is continuous on $\mathcal{P}$.

\section{Smooth Polygons and their Homomorphisms}

The word 'smooth' will be used as a synonym for $k$-times continuously differentiable, for some $k$ with $2 \leq k \leq \infty$.

(4.1) DEFINITION. Let $\mathfrak{P}=(\mathcal{P}, \mathcal{L}, \mathcal{F})$ be a topological polygon. If $\mathcal{P}$ and $\mathcal{L}$ are smooth manifolds of positive dimension, then by [24] the spaces $\mathcal{P}$ and $\mathcal{L}$ are compact and connected. By Grundhöfer and Van Maldeghem [14, 2.1(b)], the domain of the map $f_{n-1}$ is open. We call the polygon $\mathfrak{P}$ smooth, if the map $f_{n-1}$ is smooth.

(4.2) PROPOSITION. Let $\mathfrak{P}=(\mathcal{P}, \mathcal{L}, \mathcal{F})$ be a smooth $n$-gon. Then every point row is a compact, connected, smooth embedded submanifold (see [23] for the case $n=3$ ).

Proof. Let $\ell$ be a line. Choose a vertex $x$ with $d(x, \ell)=n$. The set $V=\{p \in$ $\mathcal{P} \mid d(p, x)=n-1\}$ is open in $\mathcal{P}$ and contains the point row $L$ corresponding to $\ell$. Now we may define a retraction $\varphi: V \rightarrow L$ by $p \mapsto f_{n-1}\left(f_{n-1}(p, x), \ell\right)$. By [6, Satz 5.13], the fixed point set $L$ of $\varphi$ is a smooth embedded submanifold.

Since the point rows and the pencils of lines are smooth submanifolds, every projectivity is a diffeomorphism, and the multiplication as well as the addition is a smooth map. Similarly, the coordinate map $\xi_{\mathbf{v}}$ is a diffeomorphism. There is an obvious generalization of (3.5) to smooth polygons.

(4.3) THEOREM. Let $K, L$ be smooth manifolds of dimension $m$ and $m^{\prime}$, respectively. Let $0_{L}, 1_{L} \in L$ and $0_{K} \in K$ be elements, and suppose that we are given smooth maps $\bullet: K \times L \rightarrow K$ and $/: K \times\left(L-\left\{0_{L}\right\}\right) \rightarrow K$ with the properties (1)-(3) of (1.7). Then $K$ is diffeomorphic to $\mathbb{R}^{m}$.

Proof. Let $E \subseteq K$ be a relatively compact, open neighborhood of $0_{K}$ in $K$ which is diffeomorphic to $\mathbb{R}^{m}$. Such a set $E$ exists, since $K$ is a smooth manifold. We shall inductively construct an increasing sequence $\left(E_{j}\right)_{j \in \mathbb{N}}$ of relatively compact, open neighborhoods of $0_{K}$, each of which is diffeomorphic to $\mathbb{R}^{m}$, such that their union is $K$. 
We put $E_{1}=E$ and $a_{1}=1_{L}$. We may choose a compatible metric $\mathrm{d}$ on $L$ in such a way that $\mathrm{d}\left(a_{1}, 0_{L}\right) \leq 1$ holds. Assume that we already have defined the sets $E_{1}, \ldots, E_{j}$. Furthermore, let $a_{1}, \ldots, a_{j} \in L$ with $\mathrm{d}\left(a_{k}, 0_{L}\right) \leq k^{-1}$ for $1 \leq k \leq j$.

To construct the set $E_{j+1}$, we first prove that there exists a neighborhood $W$ of $0_{L}$ such that $\overline{E_{j}} \bullet W$ is contained in $E_{j}$. Since the multiplication $\bullet$ is continuous, we find for every element $e \in \overline{E_{j}}$ a neighborhood $W_{e}$ of $0_{L}$ and a neighborhood $U_{e}$ of $e$ with the property that the inclusion $U_{e} \bullet W_{e} \subseteq E_{j}$ holds. Since $E_{j}$ is compact, we find a finite subcover $\left\{U_{1}, \ldots, U_{n}\right\}$ of $\left\{U_{e} \mid e \in \overline{E_{j}}\right\}$. Let $\left\{W_{1}, \ldots, W_{n}\right\}$ be the corresponding subfamily of $\left\{W_{e} \mid e \in \overline{E_{j}}\right\}$, and set $W=\cap_{k=1}^{n} W_{k}$. Then, by construction, we have $\overline{E_{j}} \bullet W \subseteq E_{j}$.

Now we choose an element $a_{j+1} \in W-\left\{0_{L}\right\}$ with $\mathrm{d}\left(a_{j+1}, 0_{L}\right) \leq 1 /(j+1)$ and we define $E_{j+1}=E_{j} / a_{j+1}$. Then we have $\overline{E_{j}} \bullet a_{j+1} \subseteq E_{j}=E_{j+1} \bullet a_{j+1}$, and since the multiplication $\bullet$ is right-cancellable by (1.7), it follows that $\overline{E_{j}} \subseteq$ $E_{j+1}$. Moreover, since the map $x \mapsto x \bullet a_{j+1}: K \rightarrow K$ is a diffeomorphism of $K$, we conclude that $E_{j+1}$ is relatively compact and open and is diffeomorphic to $\mathbb{R}^{m}$

Having constructed the sequence of $m$-cells $\left(E_{j}\right)_{j \in \mathbb{N}}$, we have to show that $\cup_{j \geq 1} E_{j}=K$. To verify this, fix an element $x \in K$. Since the associated sequence $\left(a_{j}\right)_{j \in \mathbb{N}}$ converges in $L$ to $0_{L}$, we can find an element $a_{j+1}$ such that $x \bullet a_{j+1} \in E_{1} \subset E_{j}$. Thus we have $x \in E_{j} / a_{j+1}=E_{j+1}$. Now, the equation $\cup_{j \geq 1} E_{j}=K$ expresses that $K$ is a regular neighborhood of $0_{K}$ in the sense of Siebenmann et al. [35]. Since $E_{1}$ is diffeomorphic to $\mathbb{R}^{m}$, it is a regular neighborhood of $0_{K}$ as well. By [35, Th. 2.1 and Section 6], all regular neighborhoods of a given point are diffeomorphic. Hence $K$ is diffeomorphic to $\mathbb{R}^{m}$.

(4.4) COROLLARY. Let $\mathfrak{P}$ be a smooth polygon. Then every point row is homeomorphic to an $m$-sphere, and every pencil of lines is homeomorphic to an $m^{\prime}$-sphere, for some integers $m, m^{\prime}>0$. Every 'punctured point row' (i.e. a point row with a point removed) is diffeomorphic to $\mathbb{R}^{m}$, and every punctured pencil of lines is diffeomorphic to $\mathbb{R}^{m^{\prime}}$ (cp. Knarr [22, 2.1]).

It is unknown whether the point rows or the pencils of lines are necessarily standard smooth spheres or not.

The fact that the point rows and the pencils of lines are manifolds implies that $n \in\{3,4,6\}$, see Knarr [22]. This result is the topological analogue of the celebrated Feit-Higman theorem [10]. There are also restrictions on the parameters $m, m^{\prime}:$ if $n=3$, then $m=m^{\prime} \in\{1,2,4,8\}$, if $n=6$, then $m=m^{\prime} \in\{1,2,4\}$, and if $n=4$ and if $m, m^{\prime}>1$, then $m=m^{\prime} \in\{2,4\}$ or $m+m^{\prime}$ is odd, see also Grundhöfer et al. [15, 1.7] and Kramer [24]. In the case of smooth projective planes, the topological spaces $\mathcal{P}$ and $\mathcal{L}$ are determined up to homeomorphism by the number $m$ (see [23]).

(4.5) COROLLARY. Let $(D, 0,1,+, \bullet)$ be a double loop (cp. Grundhöfer and 
Salzmann [16] or Hofmann and Strambach [19]). Suppose that $D$ is a smooth manifold of positive dimension $m$. Assume moreover that $\bullet: D \times D \rightarrow D$ is a smooth map, and that the solution $x$ of $x \bullet a=b$ depends smoothly on $(a, b) \in$ $(D-\{0\}) \times D$. Then $D$ is diffeomorphic to $\mathbb{R}^{m}$, and $m \in\{1,2,4,8\}$ by Adams' result [1, Th. 1.1.1].

(4.6) DEFINITION. A homomorphism $\varphi$ between two smooth $n$-gons is called smooth if $\varphi$ is smooth on $\mathcal{P}$ and $\mathcal{L}$ and if the images of $\mathcal{P}$ and $\mathcal{L}$ are smooth submanifolds.

(4.7) THEOREM. Every continuous homomorphism $\varphi: \mathfrak{P} \rightarrow \mathfrak{P}^{\prime}$ between smooth polygons is a smooth embedding. (See Betten [2, Satz 2] for the case of collineations of smooth two-dimensional projective planes.)

Proof. By (3.4), the map $\varphi$ is a topological embedding. The restriction of $\varphi$ to any point row and to any pencil of lines is a smooth embedding:

Let $v_{0} \in \mathcal{V}$ be a vertex. Fix an ordinary $n$-gon $\mathbf{v}=\left(v_{0}, v_{1}, \ldots, v_{2 n-1}, v_{0}\right)$ and a vertex $a$ in $\mathfrak{P}$ as in (1.8). Put $K=\mathcal{V}_{v_{0}}-\left\{\infty_{K}\right\}$ and $K^{\prime}=\mathcal{V}_{\varphi\left(v_{0}\right)}^{\prime}-\left\{\varphi\left(\infty_{K}\right)\right\}$. Thus we get a continuous, injective homomorphism of the smooth right loop $K$ into the smooth right loop $K^{\prime}$. By [3], the restriction $\varphi \mid K$ is an immersion. Replacing $v_{i}$ by $v_{2 n-i}$, we obtain that $\varphi \mid \mathcal{V}_{v_{0}}$ is an immersion.

By (2.7), the restriction of $\varphi$ to any big cell is a smooth immersion. Hence $\varphi$ is an injective immersion, and in fact an embedding, because $\mathfrak{P}$ is compact.

(4.8) COROLLARY. A topological polygon $\mathfrak{P}$ possesses at most one differentiable structure which makes it into a smooth polygon.

(4.9) COROLLARY. The group of all continuous automorphisms of a smooth polygon $\mathfrak{P}=(\mathcal{P}, \mathcal{L}, \mathcal{F})$ is a smooth Lie transformation group on $\mathcal{P}, \mathcal{L}$ and $\mathcal{F}$, with respect to the compact-open topology.

Proof. The group of all continuous automorphisms is locally compact by Burns and Spatzier [7, 2.1], see Grundhöfer [12] and Salzmann [32] for $n=3$. Being a locally compact group of diffeomorphisms, it is a smooth Lie transformation group (cp. Montgomery and Zippin [25, pp. 208, 212]).

\section{Strong Inhomogeneity of Certain Isoparametric Hypersurfaces}

In this section, we apply our results about smooth polygons to prove a strong inhomogeneity result for certain isoparametric hypersurfaces. For basic information on isoparametric hypersurfaces see the paper by Ferus et al. [17], as well as the book by Cecil and Ryan [8].

(5.1) DEFINITION. Let $\mathcal{F} \subseteq \mathbb{S}^{r}$ be a complete isoparametric hypersurface with $g \geq 3$ distinct principal curvatures. Let $\mathcal{P}, \mathcal{L}$ denote the two focal submanifolds of $\mathcal{F}$ with the canonical projections $\pi_{1}: \mathcal{F} \rightarrow \mathcal{P}$ and $\pi_{2}: \mathcal{F} \rightarrow \mathcal{L}$. We may embed $\mathcal{F}$ into $\mathcal{P} \times \mathcal{L}$ by means of the map $x \mapsto\left(\pi_{1}(x), \pi_{2}(x)\right)$, and hence we may consider the triple $(\mathcal{P}, \mathcal{L}, \mathcal{F} \hookrightarrow \mathcal{P} \times \mathcal{L})$ as an incidence structure. 
Ferus et al. [17] constructed a large class of isoparametric hypersurfaces with $g=4$ distinct principal curvatures as follows: let $P_{0}, P_{1}, \ldots, P_{m} \in \mathbb{R}^{2 k \times 2 k}$ be symmetric matrices satisfying the identities $P_{i} P_{j}+P_{j} P_{i}=2 \delta_{i j} 11$. Put $H(x)=\Sigma_{i=0}^{m}\left\langle P_{i} x, x\right\rangle^{2}$. The set

$$
\mathcal{F}=\left\{x \in \mathbb{S}^{2 k-1} \mid H(x)=\frac{1}{2}\right\}
$$

is an isoparametric hypersurface with $g=4$ distinct principal curvatures, provided that $m, k-m-1>0$. The focal sets are given by $\mathcal{P}=\left\{x \in \mathbb{S}^{2 k-1} \mid H(x)=0\right\}$ and $\mathcal{L}=\left\{x \in \mathbb{S}^{2 k-1} \mid H(x)=1\right\}$, respectively. We denote the corresponding incidence structure by $\mathfrak{P}\left(P_{0}, \ldots, P_{m}\right)$, and we call $\mathcal{F}$ an isoparametric hypersurface of FKM-type.

The following result is due to Thorbergsson [39]:

(5.2) THEOREM. The incidence structure $\mathfrak{P}\left(P_{0}, \ldots, P_{m}\right)$ is a smooth generalized quadrangle. The point rows are spheres of dimension $k-m-1$, and the pencils of lines are spheres of dimension $m$.

Let $\mathfrak{P}=(\mathcal{P}, \mathcal{L}, \mathcal{F})$ be a smooth generalized polygon. To each point $p \in \mathcal{P}$ we may attach the number

$$
\left.d(p)=\operatorname{codim}\left\langle T_{p} L\right| L \text { point row through } p\right\rangle,
$$

where $T_{p} L$ denotes the subspace of the tangent space $T_{p} \mathcal{P}$ consisting of all vectors tangent to the point row $L$. By (4.7), every continuous automorphism of $\mathfrak{P}$ preserves the number $d$.

(5.3) THEOREM. Let $\mathfrak{P}\left(P_{0}, \ldots, P_{m}\right)$ be a smooth quadrangle associated to an isoparametric hypersurface $\mathcal{F}$ of $F K M$-type. If $m \equiv 3(4)$, then the group $\Sigma$ of all continuous automorphisms is not transitive on the point space $\mathcal{P}$.

Proof. Let $v \in N_{p} \mathcal{P}$ be a unit normal vector of $\mathcal{P} \subseteq \mathbb{S}^{2 k-1}$. The shape operator $S_{v}$ has eigenvalues $0, \pm 1([17,4.5])$, and the kernel of $\left(S_{v}-\mathbb{1 1}\right)$ is precisely the tangent space $T_{p} L$ of the point row $L$ determined by the line $\ell=\exp _{v}(\pi / 4) \in \mathcal{L}$. It is shown in $[17,5.8]$ that the number $d(p)$ is not constant on $\mathcal{P}$, provided that $m \equiv 3(4)$. Thus, by the above remark, the group $\Sigma$ cannot be transitive on $\mathcal{P}$.

Note that $\Sigma$ is precisely the group of all $\pi_{1}, \pi_{2}$-equivariant homeomorphisms of $\mathcal{F}$. Due to the rigidity of isoparametric hypersurfaces (2.7 in [17]), the isometry group $I(\mathcal{F})$ is a (compact) subgroup of $\Sigma$. In general (e.g. in the case of Moufang quadrangles), the group $\Sigma$ itself is non-compact and thus strictly larger than the isometry group $I(\mathcal{F})$.

\section{Acknowledgement}

We would like to thank T. Grundhöfer and B. Mühlherr for providing us with tea and cookies. 


\section{References}

1. Adams, J. F.: On the non-existence of elements of Hopf invariant one, Ann. Math. 72 (1960), 20-104.

2. Betten, D.: 2-dimensionale differenzierbare projektive Ebenen, Arch. Math. 22 (1971), 304-309.

3. Bödi, R. and Kramer, L.: Differentiability of continuous homomorphisms between smooth loops, Results Math. 25 (1994), 13-19.

4. Breitsprecher, S.: Uniforme projektive Ebenen, Math. Z. 95 (1967), 139-168.

5. Breitsprecher, S.: Projektive Ebenen, die Mannigfaltigkeiten sind, Math. Z. 121 (1971), 157-174.

6. Bröcker, Th. and Jänich, K.: Einfuihrung in die Differentialtopologie, Springer, Berlin, 1973.

7. Burns, K. and Spatzier, R.: On topological Tits buildings and their classification, Publ. Math. IHES 65 (1987), 5-34.

8. Cecil, T. E. and Ryan, P. J.: Tight and Taut Immersions of Manifolds, Res. Notes in Math. 107, Pitman, Boston, 1985.

9. Dembowski, P.: Homomorphismen von $\lambda$-Ebenen, Arch. Math. 10 (1959), 46-50.

10. Feit, W. and Higman, G.: The nonexistence of certain generalized polygons, J. Algebra 1 (1964), $114-131$.

11. Forst, M.: Topologische 4-gone, Mitt. Math. Sem. Giessen 147 (1981), 65-129.

12. Grundhöfer, T.: Automorphism groups of compact projective planes, Geom. Dedicata 21 (1986), 291-298.

13. Grundhöfer, T. and Knarr, N.: Topology in generalized quadrangles, Topology Appl. 34 (1990), 139-152.

14. Grundhöfer, T. and Van Maldeghem, H.: Topological polygons and affine buildings of rank three, Atti. Sem. Mat. Fis. Univ. Modena 38 (1990), 459-479.

15. Grundhöfer, T., Knarr, N. and Kramer, L.: Flag-homogenous compact connected polygons, Geom. Dedicata 55(1) (1995), 95-114.

16. Grundhöfer, T. and Salzmann, H.: Locally compact double loops and ternary fields, in O. Chein, H. D. Pflugfelder and J. D. H. Smith (eds) Quasigroups and Loops: Theory and Applications, Heldermann, Berlin, 1990.

17. Ferus, D., Karcher, H. and Münzner, H.-F.: Cliffordalgebren und neue isoparametrische Hyperflächen, Math. Z. 177 (1981), 479-502.

18. Hanssens, G. and Van Maldeghem, H.: Coordinatization of generalized quadrangles, Ann. Discr. Math. 37 (1988), 195-208.

19. Hofmann, K. H. and Strambach, K.: Topological and analytical loops, in O. Chein, H. D. Pflugfelder, and J. D. H. Smith (eds) Quasigroups and Loops: Theory and Applications, Heldermann, Berlin, 1990.

20. Hughes, D. R.: On homomorphisms of projective planes, AMS Proc. Symp. Appl. Math. 10 (1960), 45-52.

21. Knarr, N.: Projectivities of generalized polygons, Ars Combin. 25B (1988), 265-275.

22. Knarr, N.: The nonexistence of certain topological polygons, Forum Math. 2 (1990), 603-612.

23. Kramer, L.: The topology of smooth projective planes, Arch. Math. 63 (1994), 85-91.

24. Kramer, L.: Compact polygons, Dissertation, Tübingen, 1994.

25. Montgomery, D. and Zippin, L.: Topological Transformation Groups, Interscience, New York, 1955.

26. Mortimer, B.: A geometric proof of a theorem of Hughes on homomorphisms of projective planes, Bull. London Math. Soc. 7 (1975), 267-268.

27. Pasini, A.: The nonexistence of proper epimorphisms of finite generalized polygons, Geom. Dedicata 15 (1984), 389-397.

28. Prieß-Crampe, S.: Angeordnete Strukturen: Gruppen, Körper, projektive Ebenen, Springer, Berlin, Heidelberg, New York, 1983.

29. Ronan, M.: Lectures on Buildings, Academic Press, San Diego, 1989.

30. Salzmann, H.: Topologische projektive Ebenen, Math. Z. 67 (1957), 436-466.

31. Salzmann, H.: Homomorphismen topologischer projektiver Ebenen, Arch. Math. 10 (1959), $51-55$.

32. Salzmann, H.: Homogene kompakte projektive Ebenen, Pacific J. Math. 60 (1975), 217-233. 
33. Salzmann, H.: Projectivities and the topology of lines, in P. Plaumann and K. Strambach (eds) Geometry - von Staudt's Point of View, Proc. NATO Adv. Study Inst., Bad Windsheim, 1980, pp. 313-337.

34. Schroth, A.: Topologische Laguerreebenen und topologische Vierecke, Dissertation, TU Braunschweig, 1992.

35. Siebenmann, L., Guillou, L. and Hähl, H.: Les voisinages ouverts réguliers, Ann. Sci. École Norm. Sup. 6 (1973), 253-293.

36. Skornjakov, L. A.: Topological projective planes (russian), Trudy Moskov. Mat. Obshch. 3 (1954), 347-373.

37. Spivak, M.: Differential Geometry Vol. I, Publish or Perish, Houston, 1979.

38. Stroppel, M.: Endomorphisms of stable planes, Sem. Sophus Lie 2 (1992), 75-81.

39. Thorbergsson, G.: Clifford algebras and polar planes, Duke Math. J. 67 (1992), 627-632.

40. Tits, J.: Buildings of Spherical Type and Finite BN-Pairs, 2nd edn, Lecture Notes in Math. 386, Springer-Verlag, Berlin, 1986. 